\title{
Inactivation of the positive LuxR-type oligomycin biosynthesis regulators OImRI and OlmRII increases avermectin production in Streptomyces avermitilis
}

\author{
YU Qing, BAI LinQuan, ZHOU XiuFen \& DENG ZiXin* \\ State Key Laboratory of Microbial Metabolism, and School of Life Sciences \& Biotechnology, Shanghai Jiao Tong University, Shanghai 200030, \\ China
}

Received July 11, 2011; accepted September 21, 2011; published online December 21, 2011

\begin{abstract}
Oligomycins are a group of 26 macrocyclic lactones that exhibit broad biological activities, including antifungal, anticancer and nematocidal activities. Analysis of the oligomycin biosynthetic gene cluster $(\mathrm{olm})$ in $S$. avermitilis revealed 2 tandem LuxR-type regulators, OlmRI (931 aa) and OlmRII (941 aa), with shared identity of 38\%. Gene replacement of olmRI or olmRII abolished oligomycin production, and this production could be partially restored in the disruptants by introducing cloned olmRI and olmRII with their native promoters, demonstrating the essential role of OlmRI and OlmRII for oligomycin biosynthesis. Quantitative real-time RT-PCR analysis revealed that transcription of 14 olm genes was differentially affected by the deletion of olmRI and olmRII. Unexpectedly, avermectin production in both mutants was enhanced at least 4-fold. The identification of the positive cluster-situated regulators, OlmRI and OlmRII, paves the way for the transcriptional analysis of oligomycin biosynthesis and for the enhancement of oligomycin and avermectin production through regulator engineering.
\end{abstract}

Streptomyces, oligomycin, regulation, avermectin, enhanced production

Citation: $\quad$ Yu Q, Bai L Q, Zhou X F, et al. Inactivation of the positive LuxR-type oligomycin biosynthesis regulators OlmRI and OlmRII increases avermectin production in Streptomyces avermitilis. Chin Sci Bull, 2012, 57: 869-876, doi: 10.1007/s11434-011-4865-5

Streptomycetes are Gram-positive soil bacteria that undergo morphological differentiations from substrate mycelia to aerial hyphae and chains of spores, and have an amazing ability to produce a great variety of valuable biologically active secondary metabolites, including hundreds of antibiotics [1]. Antibiotics are usually produced during the late growth phase, and the coordinated expression of all the genes in an antibiotic biosynthetic gene cluster is tightly regulated in response to environmental conditions through a complex regulatory network [2].

The regulatory hierarchies employed by some representative biosynthetic pathways have been elucidated, and a great number of regulatory genes involved in antibiotic production and morphological differentiation have been identified [3]. These genes take part in the regulation at dif-

*Corresponding author (email: zxdeng@ @stu.edu.cn) ferent levels to respond to various external environmental changes and to the internal growth-phase transition. Some pleiotropic regulators execute global functions not only in secondary metabolism, but also in morphological differentiation [4]. bldA, encoding a tRNA recognizing the rare leucine codon UUA, is such a regulator. Mutation of bldA causes loss of aerial hyphae and of actinorhodin and undecylprodigiosin production [5-7]. Other important regulatory cascades are switched on by hormone-like molecules, such as the A-factor of S. griseus [8] or $\gamma$-butyrolactone in $S$. virginiae $[9,10]$. In $S$. griseus, A-factor binds to the A-factor receptor protein, ArpA, and initiates the transcription of $a d p A$ by dissociating the DNA-bound ArpA from the $a d p A$ promoter. AdpA then plays a central regulatory role in morphological development and secondary metabolism pathways by binding the transcriptional start sites of target genes and recruiting RNA polymerase to the promoter re- 
gions. In contrast, pathway-specific regulators only affect the transcription of a single antibiotic biosynthetic pathway, within which the genes are clustered. However, clustersituated regulators, which are considered to be pathway-specific, have also been shown to influence colony development [11].

Some well-known pathway-specific regulators situated in Type I polyketide synthase gene clusters belong to a novel LAL family (large ATP-binding regulators of the LuxR family) of activators [12]. They are large proteins that feature an N-terminal ATP/GTP-binding domain containing the Walker A and B motifs [13], and a C-terminal LuxR family helix-turn-helix (HTH) DNA-binding domain [14]. Examples of LAL transcriptional activators include PikD, required for pikromycin production by $S$. venezuelae [15], NysRI and NysRIII for nystatin biosynthesis in $S$. noursei [16], GdmRI and GdmRII for geldanamycin biosynthesis in S. hygroscopicus 17997 [17], and PimR for pimaricin biosynthesis in S. natalensis [18]. In particular, the LAL family regulator, AveR, is not only the key pathway-specific activator for avermectin biosynthesis but also negatively affects oligomycin production by down-regulating olmRI and olmRII transcription [19].

Oligomycins are a group of 26 macrolides that exhibit antifungal, antitumor and nematocidal activities [20-22]. They act as inhibitors of mitochondrial F1F0-ATPase leading to ATP depletion [23]. They can induce apoptosis in a variety of cell types, make cells more susceptible to cell death and can also cause a switch in the death mode from apoptosis to necrosis [24,25]. Recently, much research has focused on the potent anti-cancer activity of oligomycin $[22,26,27]$. The genome of $S$. avermitilis ATCC 31267, has been sequenced and contains more than 20 secondary metabolite biosynthetic clusters [28]. Oligomycin A and avermectin are the two main antibiotics synthesized by $S$. avermitilis ATCC 31267 [29]. The oligomycin biosynthetic gene cluster extends to about $90 \mathrm{~kb}$, including seven long open reading frames (ORFs) encoding multifunctional Type I polyketide synthases (PKSs) (Figure 1 from [30]).

Genes olmRI (GI15823972) and olmRII (GI15823973) of S. avermitilis ATCC 31272, located upstream of the first PKS gene $\operatorname{olmAl}$, were predicted to be LuxR-family regulators. Through gene inactivation and complementation followed by transcriptional analysis, we describe here the in- volvement of olmRI and olmRII in oligomycin biosynthesis and their influence on avermectin production.

\section{Materials and methods}

\subsection{Bacterial strains, media, growth conditions, DNA manipulation and sequence analysis}

All strains and plasmids used in this study are listed in Table S1. Culture and standard bacteriological methods were performed as described by Sambrook et al. [31] and Kieser et al. [32]. According to refs. [33,34], the wild-type strain, $S$. avermitilis ATCC 31272, represents a lyophilized culture obtained by ultraviolet irradiation of $S$. avermitilis ATCC 31267. The avermectin and oligomycin production, and the DNA sequence in the oligomycin cluster, were confirmed in this study to be consistent between the two strains. E. coli strains were grown at $37^{\circ} \mathrm{C}$ in Luria-Bertani medium. $S$. avermitilis ATCC 31272 and its mutants were grown at $30^{\circ} \mathrm{C}$ on SFM [32] agar for oligomycin fermentation and for conjugation with E. coli ET12567/pUZ8002. YD agar [35] was used for sporulation, and TSBY liquid medium [32] supplemented with $10.3 \%(\mathrm{w} / \mathrm{v})$ sucrose was used for mycelial growth. For Streptomyces, apramycin and thiostrepton were respectively used at 30 and $20 \mu \mathrm{g} / \mathrm{mL}$ in agar plates and at 15 and $10 \mu \mathrm{g} / \mathrm{mL}$ in liquid medium.

General techniques for DNA manipulation were used as described in refs. [31,32]. Synthesis of oligonucleotide primers and DNA sequencing of PCR products were performed by Invitrogen Biotechnology (China). Restriction digestions were carried out according to the protocols for the enzymes (Fermentas Inc., Canada). Analyses of amino acid sequences were performed using Blastp and ClustalW, and Pfam at http://pfam.janelia.org/ [36].

\subsection{Inactivation of olmRI and olmRII and complemen- tation of the mutants}

Fragments $(7.1-7.3 \mathrm{~kb})$ were recovered from $\operatorname{BamH} \mathrm{I}-$ digested total genomic DNA separated by agarose gel electrophoresis, and cloned into pBluescript $\mathrm{SK}(+)$. After screening clones by $P v u$ II and Xho I digestion, a plasmid containing the 7266-bp olmRI-olmRII fragment was confirmed and named pJTU1714. The fragment was then

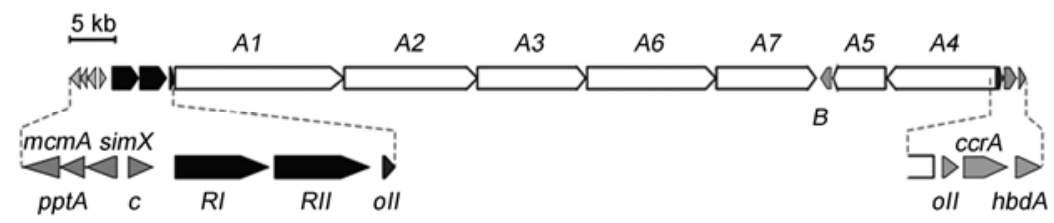

Figure 1 Oligomycin biosynthetic gene cluster of $S$. avermitilis. Arrows indicate ORFs and the orientation of transcription. RI and RII (black arrows, olmRI and olmRII), LAL-family regulators; A1-A7 (blank arrow boxes, olmA1-olmA7), type I polyketide synthases. The grey arrows indicate the post-modification genes: $m c m A$, methylmalonyl-CoA mutase; pptA, phosphopantetheinyl transferase; $\operatorname{simX}$, simX4 homolog; $C$ (olmC), thioesterase; $B$ $($ olmB), cytochrome P450; OII (olmOII), hypothetical protein; $c c r A$, crotonyl-CoA reductase; $h b d A$, 3-hydroxyacyl-CoA dehydrogenase. 
BamH I-digested and transferred to pJTU412 to generate plasmid pJTU1715. To inactivate olmRI and olmRII, two aac(3)IV-oriT cassettes were amplified with the template pIJ773, using the primers olmRI-tgtP1 \& P2, and olmRIItgtP3 \& P4 respectively (Table S2). They were then separately introduced into $E$. coli BW25113/pIJ790/ pJTU1715 by electroporation and recombined with pJTU1715 under temperature pressure [37] to generate the apramycinresistant disruptants, pJTU1710 for $\Delta$ olmRI and pJTU1727 for $\Delta$ olmRII. pJTU1710 and pJTU1727 were individually conjugated into $S$. avermitilis ATCC 31272 as described [32]. Apramycin-resistant thiostrepton-sensitive $\left(\mathrm{Am}^{\mathrm{R}} \mathrm{Tsr}^{\mathrm{S}}\right)$ double-crossover exconjugants YQ6 $(\Delta o l m R I)$ and YQ18 $(\Delta o l m R I I)$ were selected and confirmed by PCR with primers olmRI-test-P1 \& P2 and olmRII-test-P3 \& P4, respectively (Table S2).

For complementation, the plasmid pJTU1739 was constructed by inserting the 7266-bp fragment from pJTU1714 into the BamH I-digested pIB139-derived vector, pJTU824 (Table S1). pJTU1739 was introduced through conjugation into both of the mutants. YQ6: pJTU1739 and YQ18: pJTU1739 were selected by an $\mathrm{Am}^{\mathrm{R}} \mathrm{Tsr}^{\mathrm{R}}$ phenotype.

\subsection{Oligomycin and avermectin production assay}

Thawed suspensions of S. avermitilis ATCC 31272 and its derivatives were inoculated into $10 \mathrm{~mL}$ TSBY liquid medium and grown at $30^{\circ} \mathrm{C}$ for $18 \mathrm{~h}$ to produce seed cultures. After detecting the wet weight of mycelia, the same amounts of seed cultures were grown on two SFM agar plates $\left(40 \mathrm{~mL}\right.$ melted medium spread to $56.5 \mathrm{~cm}^{2}$ ) at $30^{\circ} \mathrm{C}$ for $72 \mathrm{~h}$ for antibiotic production. The solid fermentation cultures were extracted twice for $8 \mathrm{~h}$ with two volumes of methanol. The methanol was evaporated and crude extracts were re-dissolved in $1 \mathrm{~mL}$ of methanol, passed through $0.22 \mu \mathrm{m}$ nylon filters, and analyzed using an Agilent 1100 series LC/MSD Trap system using a ZORBAX Extend-C18 column $(5 \mu \mathrm{m}, 2.1 \mathrm{~mm} \times 150 \mathrm{~mm}$, Agilent, USA). Eluent A was Milli-Q (Millipore, USA) deionized water with $0.2 \%$ formic acid, and eluent $\mathrm{B}$ was acetonitrile. The $\mathrm{LC}$ was monitored at $230 \mathrm{~nm}$ and the flow rate was at $0.27 \mathrm{~mL} \mathrm{~min}^{-1}$ using a gradient mobile phase starting with $60 \% \mathrm{~B}(\mathrm{v} / \mathrm{v})$ and ending with $80 \% \mathrm{~B}$ for $15 \mathrm{~min}$, followed by the isocratic mobile phase containing $80 \% \mathrm{~B}$ for $2 \mathrm{~min}$, and finally by an increasing gradient phase ending with $90 \%$ B for $10 \mathrm{~min}$. Oligomycin A from $S$. diastatochromogenes (SigmaAldrich Corp., Saint Louis, MO, USA) was used as the standard with a retention time of $15.3 \mathrm{~min}$.

1.4 Isolation of total RNA and gene expression analysis by quantitative real-time reverse transcriptase PCR (qPCR)

For RNA isolation, S. avermitilis was grown for 3-4 d at $30^{\circ} \mathrm{C}$ on SFM agar plates covered with cellophane sheets.
Mycelia were scraped from the cellophane and disrupted in a Precellys Homogenizer $(5500 \times g, 1 \times 30 \mathrm{~s}$; Peqlab, Germany) with glass beads (150-212 $\mu \mathrm{m}$, Sigma). RNA was isolated with the total RNA Isolation Kit from SBS Genetech and treated with DNase I (RNAase-free, Fermentas) to eliminate genomic DNA.

RNA $(3 \mu \mathrm{g})$ was used to generate cDNA using RevertAid $^{\mathrm{TM}} \mathrm{H}$ Minus Reverse Transcriptase and Random Hexamer Primer (Fermentas) in $20-\mu \mathrm{L}$ reactions, as described in the manufacturer's protocol. The synthesized cDNA $(0.6 \mu \mathrm{L})$ was used as template for each reaction using the primers listed in Table S3. The sigma factor gene $h r d B$ and the 16S rRNA gene were used as references and the primers were designed according to the 16S rRNA gene of $S$. lividans (X86354.1) and the hrdB gene of $S$. coelicolor (X52983.1), respectively. The primers for the target genes within the oligomycin cluster were designed to match the sequence of the $S$. avermitilis oligomycin biosynthetic gene cluster (AB070940.1, GI15823967). The qPCR reaction mixtures were prepared according to the protocol of Maxi$\mathrm{ma}^{\circledR}$ SYBR Green/ROX qPCR Master Mix (Fermentas). The qPCR assays were carried out in triplicate using the Applied Biosystems 7500 Fast Real-Time PCR System (Applied Biosystems Inc., USA) and then repeated 3 times with RNA isolated from independent batches. For a given target gene, the relative transcript copy number was the ratio of its transcribed copies in mutant samples to its copies in the wild-type control. The ratio was calculated using the comparative $C_{\mathrm{t}}$ method with the formula $2^{-\Delta \Delta C_{\mathrm{t}}}\left(\Delta \Delta C_{\mathrm{t}}=\right.$ $\Delta C_{\mathrm{t} \text { sample (target-reference) }}-\Delta C_{\mathrm{t}}$ control (target-reference) ) [38]. All the key genes involved in oligomycin biosynthesis were analyzed. For each gene, the transcript copy number in the control wild-type strain was assigned a value of 1.0. A value higher than 2, suggested that the transcription of a target gene was distinctly activated or increased in the mutant. A value lower than 0.5 showed decreased or depressed transcription.

\section{Results and discussion}

\subsection{In silico analysis of regulatory genes olmRI and olmRII}

Two putative regulatory genes, olmRI and olmRII, are located upstream of $\operatorname{olmA1}$, the first Type I PKS gene of the oligomycin biosynthesis pathway in $S$. avermitilis ATCC 31272 [30]. Blastp analysis revealed that OlmRI (931 amino acids) and OlmRII (943 amino acids) share 38\% identity, and are putative members of the LAL family (large ATP-binding regulators of the LuxR family) of transcriptional activators [12]. Multiple alignments of LAL regulators in the polyketide biosynthesis pathway revealed conserved motifs that are also contained in OlmRI and OlmRII (Figure 2(a)). Walker A and B motifs are present at the $\mathrm{N}$ termini (Figure 2(b)) and LuxR-type HTH DNA binding motifs [14] are located at the $\mathrm{C}$ termini (Figure 2(c)). Close 
to the HTH motifs are tetratricopeptide repeats (TPR) [39] at amino acid residues $615-834$ in OlmRI and at 687-821 in OlmRII (Figure 2(a)).

The Walker A and B motifs of OlmRI and OlmRII are characteristic of the triphosphate-binding domain of a large family of ATPases associated with diverse cellular activities. The two regulators were therefore proposed to hydrolyze NTP to activate oligomycin biosynthesis. Interestingly, Walker A motifs in OlmRI (aa 32-39) and OlmRII (aa 17-24) have a truncated core motif GXGK(R)T(S), compared to the consensus ATP-binding sequence, GXXGXGKT [13]. The native $\mathrm{S} 32_{\mathrm{OlmRI}}$ and $\mathrm{A} 17_{\text {OlmRII }}$ are different from the highly conserved glycine residue of other well-known Walker A motifs (indicated by a grey circle in Figure 2(b)). This reveals the diversity of the conserved sequences of the functional Walker A core motifs. The two regulators also have TPR domains implicating protein-protein interactions and the C-terminal LuxR-family DNA-binding helix-turn-helix domains. The known LuxRfamily proteins, such as NysRI, NysRIII and PimR, can activate the biosynthesis of macrocyclic lactones. It was thus expected that inactivation of olmRI or olmRII would reduce or abolish oligomycin production by reducing the transcription of the cognate genes, which specify the enzymes involved in oligomycin biosynthesis.

\subsection{OlmRI is essential for oligomycin production}

To ascertain the involvement of olmRI in oligomycin biosynthesis, $300 \mathrm{bp}$ of the coding region of olmRI was replaced by an aac(3)IV-oriT cassette using Redirect Technology [37] (Figure 3(a)). Six apramycin-resistant and thiostrepton-sensitive $\left(\mathrm{Am}^{\mathrm{R}} \mathrm{Tsr}^{\mathrm{S}}\right)$ mutants were confirmed by PCR amplification using primers olmRI-test-P1 and $\mathrm{P} 2$ and named as YQ6. The wild type gave a $1.16-\mathrm{kb}$ amplified fragment, whereas YQ6 produced a 2.29-kb amplicon (Figure 3(b)).

By comparing the size and morphological features of the single colonies on MM and SFM agar, the growth rate and morphology of wild type and YQ6 were exactly alike. We determined oligomycin yields of the wild type and mutant after $120 \mathrm{~h}$ of growth on SFM plates at $30^{\circ} \mathrm{C}$ when oligomycin production was maximal. In two independent mutants, HPLC profiles did not show any detectable peak corresponding to oligomycin (Figure 3(c)). Complemented with the plasmid pJTU1739, the oligomycin productivity of YQ6 was restored to about $10 \%$ of the wild-type level. Therefore, olmRI was found to execute positive control on oligomycin biosynthesis.

\subsection{Inactivation of olmRII abolished oligomycin pro- duction}

Using a similar strategy as above, a 2040-bp internal region of olmRII was replaced by the aac(3)IV-oriT cassette (Figure 4(a)). Nine $\mathrm{Am}^{\mathrm{R}} \mathrm{Tsr}^{\mathrm{S}}$ mutants were verified by PCR
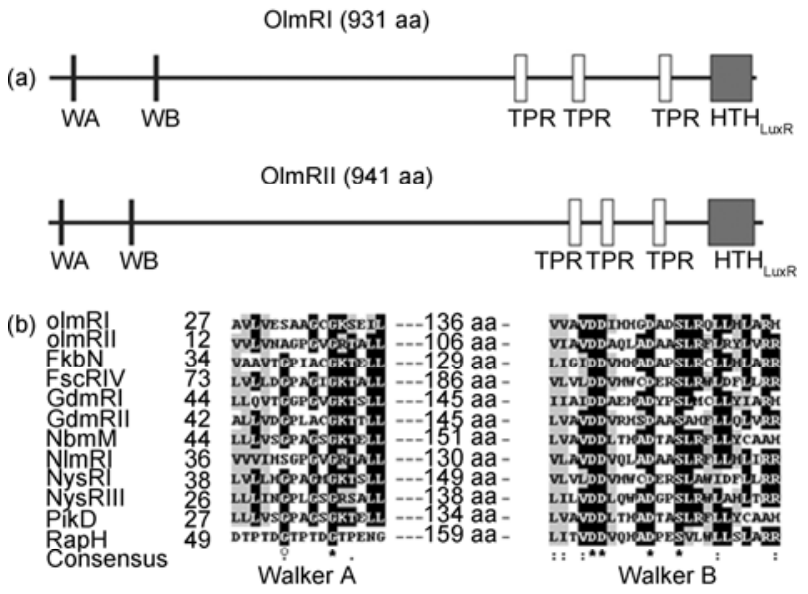

$\begin{array}{ll}\text { (c) olmRI } & 871 \\ \text { olmR II } & 867 \\ \text { FkbN } & 855 \\ \text { FscRIV } & 928 \\ \text { GdmRI } & 896 \\ \text { GdmR II } & 864 \\ \text { NbmM } & 883 \\ \text { NImRI } & 865 \\ \text { NySRI } & 890 \\ \text { NysRIII } & 870 \\ \text { PikD } & 866 \\ \text { RapH } & 886 \\ \text { Consensus } & \end{array}$

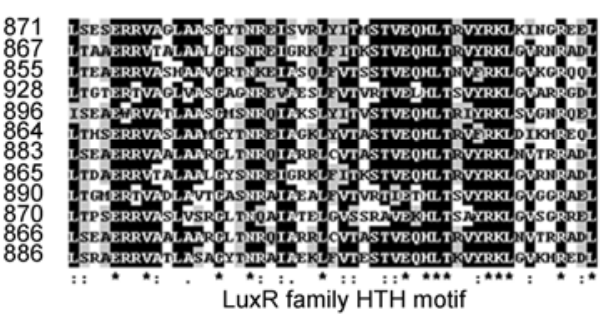

Figure 2 Structures and alignment of the OlmRI and OlmRII regulators. (a) Conserved motifs in OlmRI and OlmRII. WA and WB, Walker A and B NTP binding motifs; TPR, tetratricopeptide repeats; $\mathrm{HTH}_{\text {LuxR }}$, LuxR-family HTH DNA-binding domains. (b) Alignment of the Walker A and B motifs from proteins resembling OlmRI and OlmRII. The circle indicates the conserved glycine residue that is divergent in OlmRI and OlmRII. The stars and spots indicate highly conserved amino acid residues. The aligned proteins include the known and putative LuxR-type regulators from various streptomyces sources: FkbN from S. hygroscopicus subsp. ascomyceticus, FscRIV from Streptomyecs sp. FR-008, GdmRI and GdmRII from S. hygroscopicus 17997, NbmM from S. narbonensis, NlmRI from $S$. nanchangensis, NysRI and NysRIII from $S$. noursei, PikD from $S$. venezuelae and RapH from S. cinnamonensis. (c) Alignment of LuxR-type HTH DNA-binding motifs.

amplification with the primers olmRII-test-P3 \& P4 and named YQ18 (Figure 4(b)).

On MM and SFM agar plates, YQ18 showed a similar growth rate and morphology to the wild type. Similar to the $\triangle$ olmRI mutant YQ6, HPLC assays showed no detectable peak corresponding to oligomycin in YQ18 (Figure 4(c)). pJTU1739 complementation of YQ18 also restored oligomycin biosynthesis, but only up to $10 \%$ of the wild-type level. Therefore, OlmRII was also identified as an activator of the LAL regulator family for oligomycin biosynthesis. Moreover, the lack of oligomycin productivity in both olmRI and olmRII mutants of S. avermitilis ATCC 31267 was also mentioned as unpublished data, without any experimental details, by Guo et al. [19].

\subsection{Transcriptional analysis of oligomycin biosynthetic genes in olmRI and olmRII mutants}

The transcription of all the key biosynthetic genes in the oligomycin cluster was analyzed by quantitative real-time 

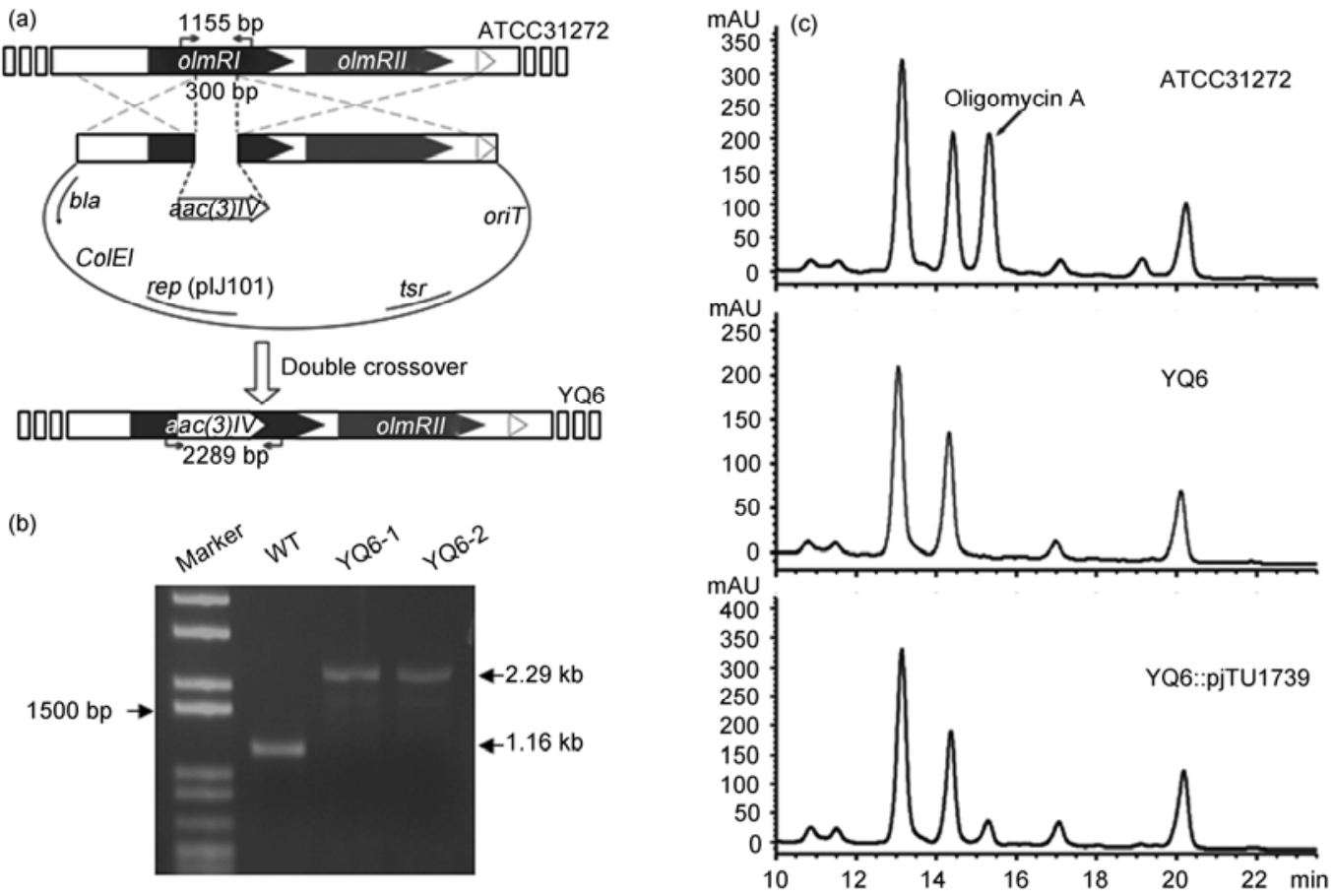

Figure 3 Inactivation of olmRI by gene replacement. (a) The strategy of generating the olmRI mutant YQ6 in S. avermitilis by replacing the 300-bp internal part of olmRI with the apramycin resistance gene $a a c(3) I V$. (b) The desired mutants were confirmed by PCR analysis using primers olmRI-test P1 \& P2 (angled arrows in (a)). (c) HPLC assay of oligomycin production. Top, extract of the wild-type ATCC31272; middle, extract from YQ6; bottom, the extract of YQ6: pJTU1739. All the other peaks represent avermectins produced by S. avermitilis.
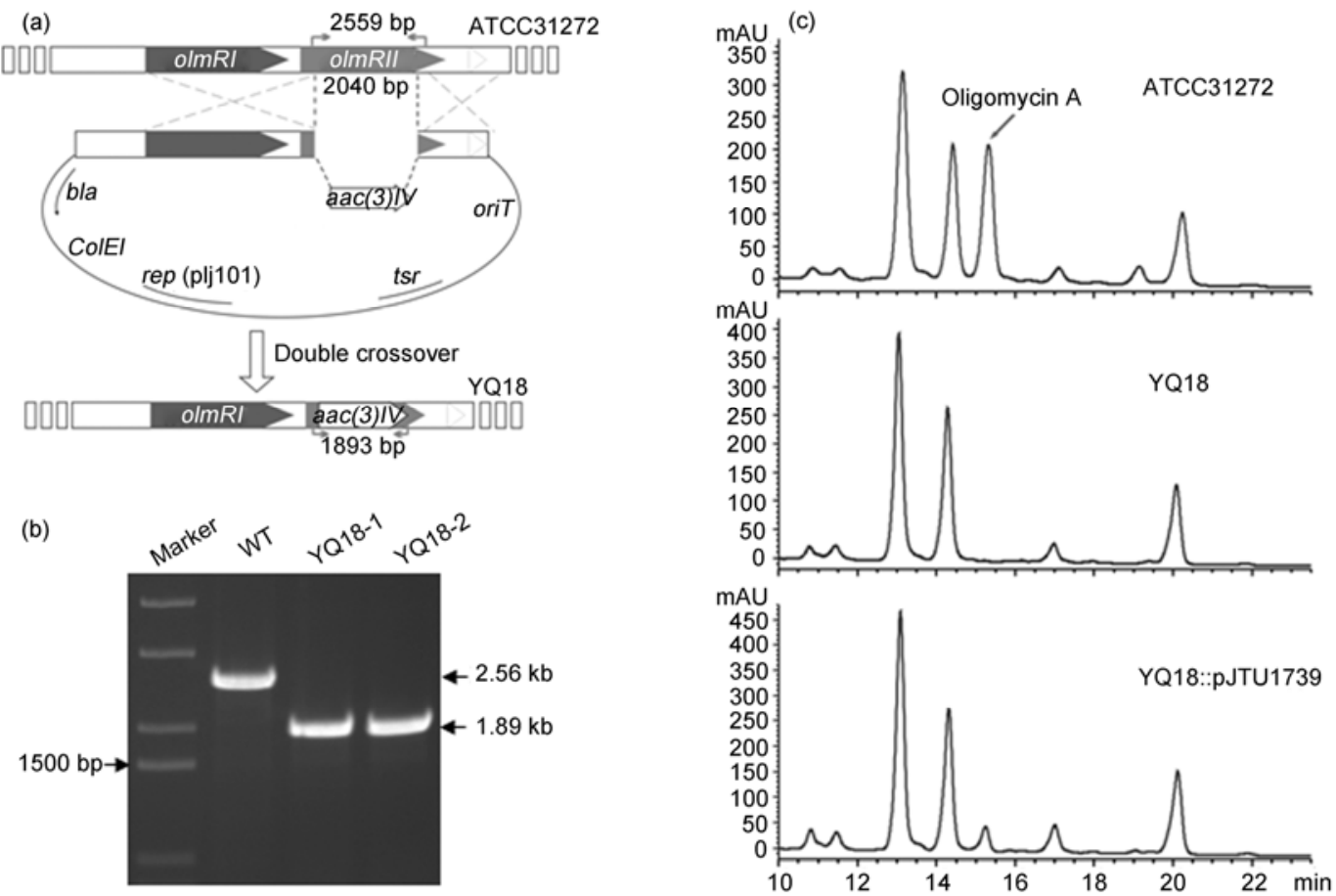

Figure 4 Inactivation of olmRII. (a) The strategy of generating olmRII mutant YQ18 in S. avermitilis. (b) The desired mutants were confirmed by PCR analysis using primers olmRI-test P3 \& P4 (angled arrows in (a)). (c) HPLC assay of oligomycin production. Top, extract of the wild-type ATCC31272; middle, extract from YQ18; bottom, the extract of YQ18: pJTU1739. All the other peaks represent avermectins. 
RT-PCR. Total RNA was isolated from the wild type, olmRI mutant YQ6 strains and olmRII mutant YQ18 strains grown for $120 \mathrm{~h}$ on SFM agar covered with cellophane. The selected key genes included PKS genes, olmA1-olmA7, cytochrome P450 gene, $\operatorname{olm} B$, the post-modification genes, olmOI, ccrA and $h b d A$, and the thioesterase gene, olmC. Moreover, $h r d B$, encoding the constitutive vegetative sigma factor and 16S rRNA gene were used as internal references. The quantitative PCR primers were specifically designed within the first $1 \mathrm{~kb}$ of each gene. The primers, qolmRI fw-rv and qolmRII fw-rv (inset in Figure 5), were designed in the regions upstream of their corresponding inserted cassettes to assess the possibility of interplay between the two regulators.

Figure 5 illustrates the general influence on the oligomycin cluster by the disruption of olmRI and olmRII. The olmRI remnant in YQ6 was transcribed to a similar extent as in the wild type. Similarly, the remnant of olmRII was transcribed at a slightly higher level than in the wild type (value 1.5-1.8). These results indicate that these two regulators did not execute auto-regulation on themselves; compared with the wild type, the transcription ratios of olmRI in YQ18 and of olmRII in YQ6 were approximately 1.2 and 1.1, respectively. This revealed no interplay between the two tandem LAL regulators.

Obviously, in YQ6, inactivation of olmRI caused a sharp reduction in the transcription of all the PKS and postmodification genes. The expression of the PKS genes, olmC and $\operatorname{olm} A 1-A 7$, located downstream and in the same orientation as the regulatory genes, was reduced to $20 \%-30 \%$ of the wild type. Expression of the genes located in the reverse orientation, olmA4-olmB, olmOI-ccrA, but not $h b d A$, was severely declined to $2 \%$ of wild type levels. In short, OlmRI is a crucial positive transcriptional regulator controlling almost the whole oligomycin biosynthetic gene cluster.
In contrast, compared to the wild type, inactivation of olmRII in YQ18 exerted different effects on olmC and olmA1-A7 transcription: olm $C$ was expressed at almost wild type levels; olmA1, the first ORF encoding the polyketide skeleton, had increased its transcription more than 2-fold; the transcription of olmA2-A7 was reduced to approximately $50 \%$. These observations indicate that when OlmRII is disrupted, OlmRI exerts very weak activation on the first four genes and strong positive control on the initial PKS genes. Meanwhile, the transcription of the reverse-direction PKS genes was nearly absent in YQ18, as in YQ6. This indicated that OlmRII was also a key positive transcriptional regulator of the reverse-direction genes in the oligomycin cluster.

Different from other homologues, structurally similar OlmRI and OlmRII are both indispensable for oligomycin biosynthesis. Bioinformatic prediction reveals a putative protein-interacting TPR motif in OlmRI and OlmRII. This motif is capable of forming homodimers or heterodimers to activate the transcription of genes essential for oligomycin biosynthesis. Because the transcription of A1 increased and decreased with the respective disruption of RII and RI, RI is demonstrated to form a homodimer to activate the first PKS gene, $A 1$. For the rest of the genes whose transcription levels were severely impaired in both YQ6 and YQ18, a heterodimer of OlmRI and OlmRII is required to function in the activation of gene transcription. Oligomycin and its derivatives are potent candidates for cancer therapy. Research on the positive regulators OlmRI and OlmRII may shed light on the over-production of this candidate compound.

\subsection{Enhanced avermectin production in both mutants}

Streptomyces avermitilis produces a series of avermectin analogues, including A2a, B1a, A1b and A1a (Figure 6(a)).

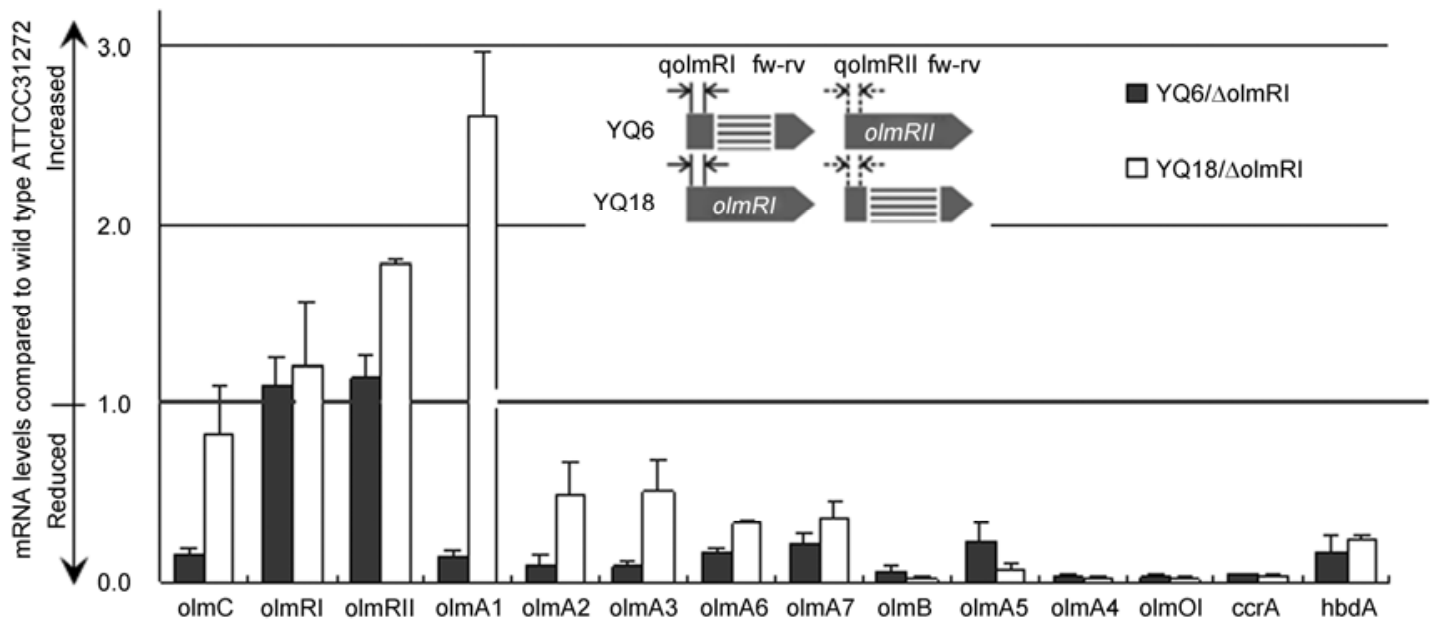

Figure 5 Transcriptional analysis of the oligomycin biosynthetic cluster using real-time RT-PCR. The histograms represent the individual target gene's transcriptional change as the ratio of the expression level in the mutants to that in the wild-type strain. Value of 1.0 (the bold line) indicates, for a certain target gene, that the mutant transcribes the same amount of mRNA as the wild type. Error bars were calculated from three independent experiments each with duplicates to validate the reproducibility. Solid bars, YQ6; white bars, YQ18. The inset shows the binding sites of the primers in olmRI and olmRII used for the quantitative real-time PCR, the solid arrow lines indicate primers qolmRI fw-rv and the dotted arrow lines indicate primers qolmRII fw-rv. The striped boxes marked the position of the replaced region in the ORFs. 

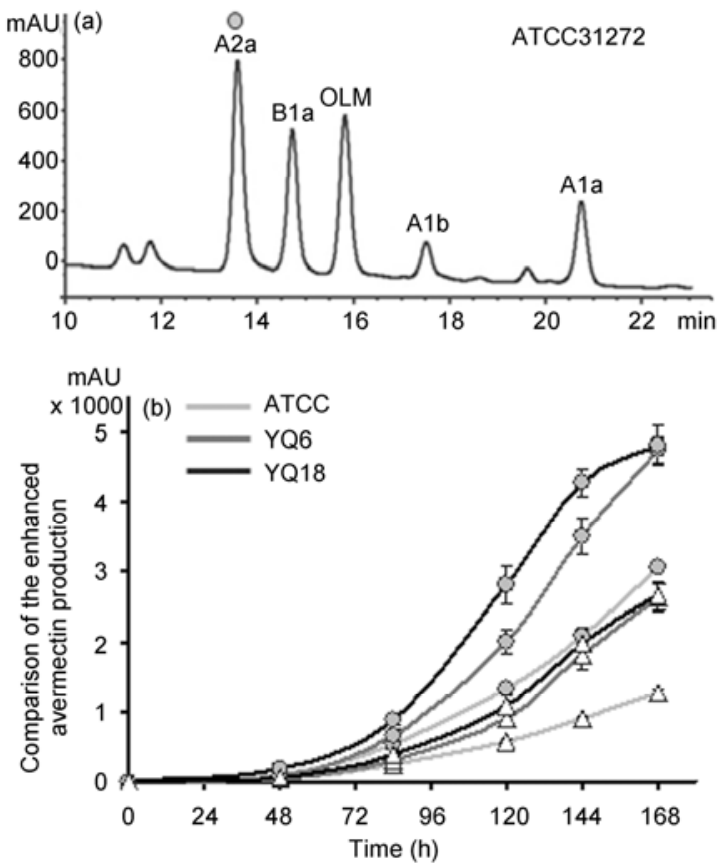

Figure 6 The avermectin production in S. avermitilis and its mutants. (a) HPLC analysis of oligomycin and the series of avermectins in wild-type $S$. avermitilis ATCC31272. A2a, B1a, A1b and A1a are the main components of the avermectins. OLM refers to the peak representing oligomycin. Corresponding to (b), A2a is marked with grey filled circles and A1b with hollow triangles. (b) The variation in antibiotic production in wild type and mutants.

In both of the LuxR-like regulator mutants, the production of avermectin was obviously enhanced. Component A2a was increased 1.5-fold in the mutants and component A1a was increased 2-fold (Figure 6(b)). However, further quantitative assays confirmed that aveR and aveA3, the known crucial regulatory and structural genes of avermectin biosynthesis, were transcribed at wild type levels (data not shown). Thus, OlmRI and OlmRII are cluster-situated specific activators of oligomycin biosynthesis. The overproduction of avermectins in the mutants may be the result of increased metabolic flux towards avermectins while the oligomycin production was switched off.

\section{Conclusion}

Using gene inactivation and complementation in S. avermitilis we have identified two positive cluster-situated regulators for oligomycin biosynthesis that show typical structural features of LuxR-type regulators, including an N-terminal ATP/GTP-binding domain and a C-terminal HTH DNAbinding domain. As detected by quantitative real-time RTPCR, the transcription of genes in the oligomycin biosynthetic cluster was altered to different extents in the olmRI or olmRII mutants, suggesting similar but different regulatory mechanisms for OlmRI and OlmRII. Moreover, even though olmRI and olmRII share high similarity and are lo- cated close to one another, the effect of one mutant on the transcription of a particular gene was not observed in the other mutant. Meanwhile, disruption of the activator genes olmRI and/or olmRII unexpectedly enhanced the production of avermectin A1a and A2a components. This not only provides understanding of the competitive utilization of substrates in Streptomyces avermitilis, but also provides a new strategy to enhance the productivity of antibiotics of interest.

The authors give their thanks to Tobias Kieser for reviewing the manuscript. This work was supported by National High Technology Research and Development Program of China (2006AA10A202), National Basic Research Program of China (2009CB118901), the National Natural Science Foundation of China (31820015), Ministry of Education of China (706021), and the Science and Technology Commission of Shanghai Municipality (075407068).

1 Hopwood D A. Streptomyces in Nature and Medicine: The Antibiotic Makers. New York: Oxford University Press, 2007

2 Bibb M. 1995 Colworth Prize Lecture. The regulation of antibiotic production in Streptomyces coelicolor A3(2). Microbiology, 1996, 142 (Pt 6): 1335-1344

3 Bibb M J. Regulation of secondary metabolism in streptomycetes. Curr Opin Microbiol, 2005, 8: 208-215

4 Champness W C, Chater K F. Regulation and integration of antibiotic production and morphological differentiation in Streptomyces spp. In: Patrick Piggot C M, Youngman P, eds. Regulation of Bacterial Differentiation. Washington D.C.: American Society for Microbiology, 1994. 61-93

5 Fernandez-Moreno M A, Caballero J L, Hopwood D A, et al. The act cluster contains regulatory and antibiotic export genes, direct targets for translational control by the bldA tRNA gene of Streptomyces. Cell, 1991, 66: 769-780

6 Takano E, Tao M, Long F, et al. A rare leucine codon in adpA is implicated in the morphological defect of bldA mutants of Streptomyces coelicolor. Mol Microbiol, 2003, 50: 475-486

7 White J, Bibb M. bldA dependence of undecylprodigiosin production in Streptomyces coelicolor A3(2) involves a pathway-specific regulatory cascade. J Bacteriol, 1997, 179: 627-633

8 Ohnishi Y, Yamazaki H, Kato J Y, et al. AdpA, a central transcriptional regulator in the A-factor regulatory cascade that leads to morphological development and secondary metabolism in Streptomyces griseus. Biosci Biotechnol Biochem, 2005, 69: 431-439

9 Kawachi R, Akashi T, Kamitani Y, et al. Identification of an AfsA homologue (BarX) from Streptomyces virginiae as a pleiotropic regulator controlling autoregulator biosynthesis, virginiamycin biosynthesis and virginiamycin M1 resistance. Mol Microbiol, 2000, 36: 302-313

10 Nakano H, Takehara E, Nihira T, et al. Gene replacement analysis of the Streptomyces virginiae barA gene encoding the butyrolactone autoregulator receptor reveals that BarA acts as a repressor in virginiamycin biosynthesis. J Bacteriol, 1998, 180: 3317-3322

11 Liu G, Tian Y, Yang H, et al. A pathway-specific transcriptional regulatory gene for nikkomycin biosynthesis in Streptomyces ansochromogenes that also influences colony development. Mol Microbiol, 2005, 55: 1855-1866

12 De Schrijver A, De Mot R. A subfamily of MalT-related ATP-dependent regulators in the LuxR family. Microbiology, 1999, 145 (Pt 6): 1287-1288

13 Walker J E, Saraste M, Runswick M J, et al. Distantly related sequences in the alpha- and beta-subunits of ATP synthase, myosin, kinases and other ATP-requiring enzymes and a common nucleotide binding fold. EMBO J, 1982, 1: 945-951

14 Henikoff S, Wallace J C, Brown J P. Finding protein similarities with nucleotide sequence databases. Methods Enzymol, 1990, 183: 111-132

15 Wilson D J, Xue Y, Reynolds K A, et al. Characterization and analy- 
sis of the PikD regulatory factor in the pikromycin biosynthetic pathway of Streptomyces venezuelae. J Bacteriol, 2001, 183: 3468-3475

16 Sekurova $\mathrm{O} \mathrm{N}$, Brautaset $\mathrm{T}$, Sletta $\mathrm{H}$, et al. In vivo analysis of the regulatory genes in the nystatin biosynthetic gene cluster of Streptomyces noursei ATCC 11455 reveals their differential control over antibiotic biosynthesis. J Bacteriol, 2004, 186: 1345-1354

17 He W, Lei J, Liu Y, et al. The LuxR family members GdmRI and GdmRII are positive regulators of geldanamycin biosynthesis in Streptomyces hygroscopicus 17997. Arch Microbiol, 2008, 189: 501-510

18 Anton N, Mendes M V, Martin J F, et al. Identification of PimR as a positive regulator of pimaricin biosynthesis in Streptomyces natalensis. J Bacteriol, 2004, 186: 2567-2575

19 Guo J, Zhao J, Li L, et al. The pathway-specific regulator AveR from Streptomyces avermitilis positively regulates avermectin production while it negatively affects oligomycin biosynthesis. Mol Genet Genomics, 2010, 283: 123-133

20 Enomoto Y, Shiomi K, Matsumoto A, et al. Isolation of a new antibiotic oligomycin G produced by Streptomyces sp. WK-6150. J Antibiot (Tokyo), 2001, 54: 308-313

21 Joseph-Horne T, Wood P M, Wood C K, et al. Characterization of a split respiratory pathway in the wheat "take-all" fungus, Gaeumannomyces graminis var. tritici. J Biol Chem, 1998, 273: 11127-11133

22 Daisuke K, Mokoto K, Kaoru Y, et al. Oligomycin SC compounds and anticancer medicine. Japan Patent, JP9208587

23 Joshi S, Cao G J, Nath C, et al. Oligomycin sensitivity conferring protein of mitochondrial ATP synthase: Deletions in the N-terminal end cause defects in interactions with $\mathrm{F} 1$, while deletions in the C-terminal end cause defects in interactions with F0. Biochemistry, 1996, 35: 12094-12103

24 Miyoshi N, Oubrahim H, Chock P B, et al. Age-dependent cell death and the role of ATP in hydrogen peroxide-induced apoptosis and necrosis. Proc Natl Acad Sci USA, 2006, 103: 1727-1731

25 Fitch M E, Chang C M, Parslow T G. The BH3 domain is required for caspase-independent cell death induced by Bax and oligomycin. Cell Death Differ, 2000, 7: 338-349

26 Kobayashi K, Nishino C, Ohya J, et al. Oligomycin E, a new antitumor antibiotic produced by Streptomyces sp. MCI-2225. J Antibiot (Tokyo), 1987, 40: 1053-1057

27 Li Y C, Fung K P, Kwok T T, et al. Mitochondria-targeting drug oli- gomycin blocked P-glycoprotein activity and triggered apoptosis in doxorubicin-resistant HepG2 cells. Chemotherapy, 2004, 50: 55-62

28 Ikeda H, Ishikawa J, Hanamoto A, et al. Complete genome sequence and comparative analysis of the industrial microorganism Streptomyces avermitilis. Nat Biotechnol, 2003, 21: 526-531

29 Ikeda H, Takada Y, Pang C H, et al. Transposon mutagenesis by Tn4560 and applications with avermectin-producing Streptomyces avermitilis. J Bacteriol, 1993, 175: 2077-2082

30 Omura S, Ikeda H, Ishikawa J, et al. Genome sequence of an industrial microorganism Streptomyces avermitilis: Deducing the ability of producing secondary metabolites. Proc Natl Acad Sci USA, 2001, 98: 12215-12220

31 Sambrook J, Fritsch E F, Maniatis T. Molecular Cloning: A Laboratory Manual. 2nd ed. Cold Spring Harbor: Cold Spring Harbor Laboratory, 1989

32 Kieser T, Bibb M J, Buttner M J, et al. Practical Streptomyces Genetics. A Laboratory Manual. Norwich, United Kingdom: John Innes Foundation, 2000

33 Albers-Schonberg G, Wallick H, Ormond R E, et al. Novel substances and process for their production. U.S. Patent, 4310519, 1982-01-12

34 Albers-Schonberg G, Wallick H, Ormond R E, et al. Strain of Streptomyces for producing antiparasitic compounds. U.S. Patent, 4429042, 1984-01-31

35 Sun Y, Zhou X, Liu J, et al. 'Streptomyces nanchangensis', a producer of the insecticidal polyether antibiotic nanchangmycin and the antiparasitic macrolide meilingmycin, contains multiple polyketide gene clusters. Microbiology, 2002, 148: 361-371

36 Finn R D, Mistry J, Tate J, et al. The Pfam protein families database. Nucleic Acids Res, 2009, 38: D211-222

37 Gust B, Challis G L, Fowler K, et al. PCR-targeted Streptomyces gene replacement identifies a protein domain needed for biosynthesis of the sesquiterpene soil odor geosmin. Proc Natl Acad Sci USA, 2003, 100: 1541-1546

38 Pfaffl M W, Horgan G W, Dempfle L. Relative expression software tool (REST) for group-wise comparison and statistical analysis of relative expression results in real-time PCR. Nucleic Acids Res, 2002, 30: e36

39 Marck C, Lefebvre O, Carles C, et al. The TFIIIB-assembling subunit of yeast transcription factor TFIIIC has both tetratricopeptide repeats and basic helix-loop-helix motifs. Proc Natl Acad Sci USA, 1993, 90: 4027-4031

Open Access This article is distributed under the terms of the Creative Commons Attribution License which permits any use, distribution, and reproduction in any medium, provided the original author(s) and source are credited.

\section{Supporting Information}

Table S1 Bacterial strains and plasmids used in this study

Table S2 Primers used for construction and confirmation of disrupted mutants

Table S3 Primers used for quantitative real-time PCR

The supporting information is available online at csb.scichina.com and www.springerlink.com. The supporting materials are published as submitted, without typesetting or editing. The responsibility for scientific accuracy and content remains entirely with the authors. 